\title{
Non-homogeneous spatial configuration of vibrissae cortical representation in layer IV of the barrel somatosensory cortex
}

\author{
ELIANA GUIC ${ }^{1}$, XIMENA CARRASCO$^{2}$, EUGENIO RODRÍGUEZ $^{1}$ \\ IGNACIO ROBLES ${ }^{3}$ and MICHAEL M MERZENICH ${ }^{4}$
}

\footnotetext{
${ }^{1}$ Department of Psychology, Faculty of Social Sciences, Pontificia Universidad Católica de Chile

${ }^{2}$ Department of Pediatrics, School of Medicine, Universidad de Chile

${ }^{3}$ Department of Surgery Hospital del Salvador, School of Medicine, Universidad de Chile (Resident)

${ }^{4}$ Keck Center for Integrative Neurosciences, UCSF, San Francisco, CA 94143, U.S.A.
}

\begin{abstract}
In the present experiments we studied exclusive and overlapping cortical representational areas of the vibrissae in layer IV cells, across the entire barrel subfield of the rat somatosensory cortex, looking for evidences that would challenge the present assumptions of homogeneity and symmetry among cortical columns in this sensorial system. Our main findings were that in layer IV of the rat barrel cortex: A) Size of vibrissae cortical representational areas $\left(X=0.4174 \mathrm{~mm}^{2} ; \mathrm{SD}=0.025\right)$ was not homogeneous, vibrissae in dorsal rows (A-B) had significantly smaller areas than those in ventral rows (D-E), a pattern that repeated itself in arcs 1-4. B) This difference arises from vibrissal representational overlap, and not from variations in exclusive zones, which are surprisingly homogeneous in size across the barrel cortex $\left(\mathrm{X}=0.079 \mathrm{~mm}^{2}\right.$; $\mathrm{SD}=0.0075)$; C) The extent of overlapping cortical areas varied systematically, with intra-row overlapping areas having a predominant bias (71.4\% of total overlapping) independent of area sizes. Accordingly, vibrissae shared receptive fields with an average of 1.15 vibrissae in the same row and 0.38 in the same arc. Barrel cortex has been viewed operationally as a conglomerate of essentially homogenous cortical columns that interact equivalently in the arc and row dimensions. Our simple but global cortical reconstructions show that this predominant view should be revised. We postulate that the vibrissae/barrels spatial disposition in rows and arcs has a relevant functional meaning, related to different sensory capabilities.
\end{abstract}

Key terms: cortical representation - barrel cortex - evoked activity - rat -somatosensory cortex - spatial configuration.

\section{INTRODUCTION}

In the Postero Medial Barrel Sub Field (PMBSF) of the somatosensory cortex, a columnar organization that is cytoarchitectonically conspicuous at the level of layer IV has been described (Woolsey \& Van der Loos, 1970). The spatial distribution of the vibrissae cortical representation parallels the somatotopic organization of the vibrissae in the snout of the rat, arranged in rows and arcs. In penetrations across the layers of a cortical column, neurons are most strongly and consistently activated by one vibrissa, the principal vibrissa (PV) (Welker, 1971). In layer IV, cells located mainly within the barrels have receptive fields comprised of only a single vibrissa, giving origin to the areas of exclusive representation of the PVs. Other layer IV neurons of the PMBSF respond to 2 or more vibrissae. These multivibrissa units, most prominent in the cortical areas between the barrels (the septa), give origin to overlapping representational areas marked by a convergence of information coming from multiple vibrissae (ArmstrongJames, 1987; Armstrong-James et al., 1992; 
Simons, 1978). It has been hypothetized that the barrel cortex may be a dual "parallel processor" using exclusive vibrissal and integrated vibrissal inputs in its processing (Ahissar et al., 2000; Brumberg et al., 1999).

The vibrissa-barrel system has become an attractive model for experimentally studying how information is processed in the cortical columns of sensory cortex, for among other reasons because it has discrete peripheral as well as cortical elements (e.g. Bretch, 2007; Ferezou et al., 2007). However, while the knowledge about barrel cortex microcircuits has reached exquisite sophistication, we cannot say the same about the understanding of the functional capabilities of this sensory system. One important assumption has been that cortical columns are essentially homogenous throughout the PMBSF and that the relationship among them is symmetrical (e.g. Miller et al., 2001; Petersen \& Diamond, 2000; Shimegi et al., 2000). There are no studies that systematically explore global functional properties across the entire barrel cortex to investigate this hypothesis.

There is significant evidence, from studies of lateral and successive signal inhibition and facilitation, that the distribution of cortical columns in rows and arcs may bear an important functional meaning (Harris et al., 2001; Simons, 78; Moore \& Schady, 2000). We also know, from the classical anatomical work of Dörfl (1982), that vibrissae of a row are coupled to each other by intrinsic muscles that have no connection to the bones. These muscles bind the base of adjacent follicles of a row, fastening them together. No equivalent coupling applies for vibrissal arcs. This functional characteristic is quite relevant, since it biases vibrissae movement's forwards and backwards, in the axes of the row in normal whisking.

An asymmetry in the system has also been observed in the plastic changes produced by follicle (Woolsey \& Wann, 1976) or cortical injury (Ito \& Seo, 1983) in neonatal rats. In both cases, the system sustains barrel rows at the expense of barrel arcs. Thus, at different system levels and with different experimental manipulations, anatomical and physiological evidence suggests that the relations among cortical columns in this system are not symmetrical.

We postulate that in the somatosensorial vibrissae-barrels system of the rat, the spatial disposition in rows and arcs, in which the peripheral (vibrissae) as well as cortical (barrels) elements are ordered, has a relevant functional meaning that is consistent with the interactions among cortical columns at the level of layer IV and that is reflected in the spatial characteristics of evoked cortical responses.

In the present experiments we studied exclusive and overlapping cortical representational areas of the vibrissae and the receptive fields of layer IV cells, across the entire PMBSF, looking for evidences that would challenge the present assumptions of homogeneity and symmetry among cortical columns in this sensorial system.

\section{METHOD}

\section{Subjects}

Naive Sprague-Dawley rats were used for the experiments. Rats were approximately 6-10 weeks old at the beginning of the study, and were housed in standard colony environment. Results from 4 rats are reported, in which the mapping of all the PMBSF was completed. All procedures involving the animals were made in accordance with the local Committee of Bioethics and the NIH Guidelines for the Care and Use of Laboratory Animals.

\section{Electrophysiological recording}

All procedures were done under aseptic conditions, and using sterile electrodes, fluids and instruments throughout. Rats were initially anesthetized with Ketamine (100 $\mathrm{mg} / \mathrm{kg}$ i.p.). The femoral vein was canulated to deliver Pentobarbital (dose of $10 \mathrm{mg} / \mathrm{kg}$ and supplements approximately every 2 hours) and physiological serum during the experiment. A medial incision was made in the ventral cervical region to carry out a tracheotomy and tracheal intubation. Rats 
were placed in a head holder. Body temperature was monitored and maintained at $37^{\circ} \mathrm{C}$ via a controlled heating blanket and rectal thermometer. The condition of the animal was also assessed by continuously monitoring EKG and by frequently checking reflexes. A medial incision in the skin of the head and neck exposed the duramater at the level of foramen magnum, which was perforated to obtain continuous drainage of cerebrospinal fluid to minimize problems of cerebral edema.

The somatosensory cortex contralateral to the stimulated mystacial pad was then exposed via a midline skin incision, retraction of temporal muscles, removal of bone using a dental drill, and a dural flap. A picture of the cortical surface vasculature was taken and the cortex maintained under a shallow well filled with liquid silicon. Tungsten microelectrodes (approximately 2 mega-ohms; Microprobe, MD) were used to record extracellular unit or multiunit potentials from a single depth corresponding to the middle cortical layers of the PMBSF. Microelectrodes were oriented normal to the pia surface and advanced through the cortex, by a micro drive equipped with a counter, to a depth of 750 microns from the piamater contact. At the beginning of the experiment we adjusted the depth of the recordings, with variations that exceptionally reached a minimum of 650 and a maximum of 850 microns in depth, until we obtained large amplitude and short latency (less than 12 $\mathrm{ms}$ ) responses driven by whisker stimulation. The following recording sites in a given experiment were usually kept at the same depth. We recorded only from one depth in each electrode track.

All vibrissae were cut at 4-5 millimeters from the skin, to enable a homogenous stimulation of different-length vibrissae. Receptive fields of neurons or small clusters of neurons were initially determined by moving individual vibrissae with a hand-held glass probe to produce just-visible movements. Later we used a controlled piezoelectric stimulator (custom made, constructed according to Simons (1983) with some variations), to stimulate those whiskers. All whiskers producing a detectable response in the recorded unit to a 100 microns deflection were included in the $\mathrm{RF}$ of the unit.

Conventional amplification, display and recording methods were used. Neural signals were amplified (differential preamplifier Grass P15-C) band pass filtered (300-5000 Hz) and displayed on an oscilloscope (Tektronik RM 502-A) and audio speaker. Overlapping representational areas were those associated with recording sites in which we recorded from multiple vibrissae, the PV being the one that produced the largest amplitude and shortest latency response. Adjacent Vibrissae (AV) were the other vibrissae that evoked responses in the same site, almost always neighbors to the PV. Exclusive areas were those in which only the PV evoked a response. Figure 1 shows all the exclusive areas recorded in one experiment and the overlapping area for C2 vibrissa. The horizontal position of each electrode penetration was recorded by reference to details of the cortical microvasculature on a magnified image of the cortical surface. In all electrophysiological mapping studies, we made electrode penetrations as close together as the vascular pattern permitted. The receptive field of the neurons recorded was carefully reconstructed, detecting all whiskers whose deflection activated a neuron.

\section{Histology}

After electrophysiological data had been collected, all animals were deeply anesthetized with Nembutal and perfused through the heart with normal saline followed by a solution of $10 \%$ formalin. The brain was removed, the PMBSF sector of cerebral cortices removed and flattened between two glass slides. The flattened cortex was placed overnight in fixative containing $30 \%$ sucrose, then frozen and sectioned at 40 microns thickness and sections were stained for Nissl substance with cresyl violet. By using a camera lucida, drawings of all the barrels were obtained for reconstruction of the barrel field; data from different slides were superimposed using the blood vessels as 
landmarks. This procedure allowed us to estimate the vertical and horizontal positioning of small electrolytic micro lesions in 3-4 sites in each rat after all the recordings were done.

\section{Areas Associated to Each Recording Site}

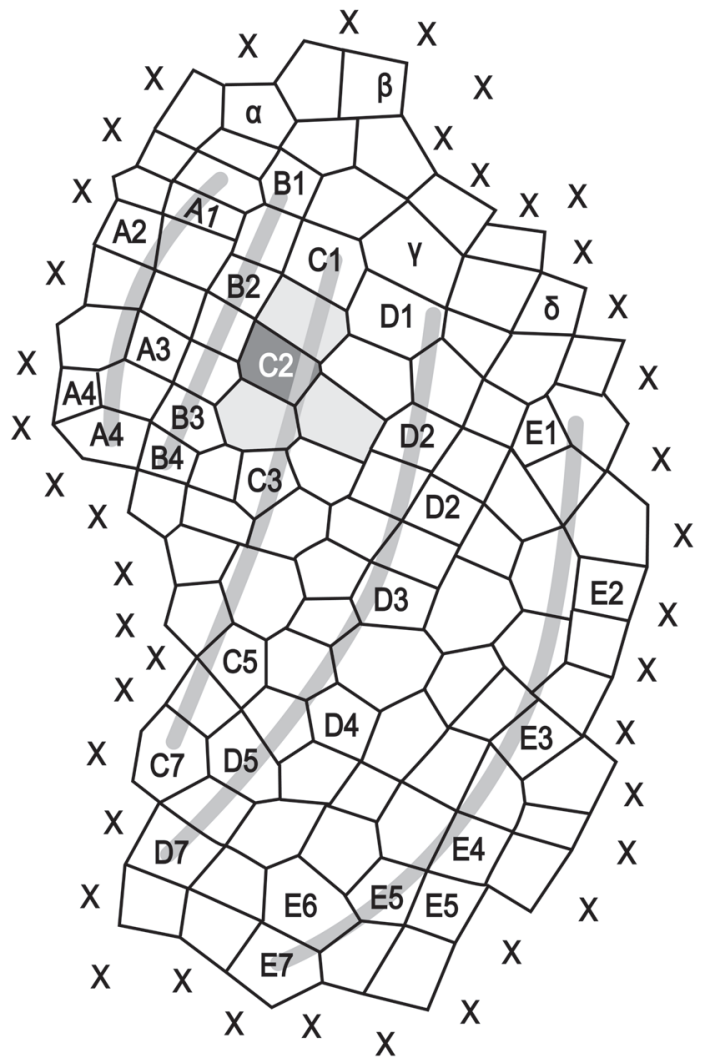

C2 Exclusive Area

C2 Overlapping Area

Figure 1: Areas associated with each recording site in a typical experiment. The crosses indicate cortical sites outside the barrel field. The matrix of areas was created by the Voronoi polygonal method (Atsuyuki et al, 2000).

The area size assigned to each recording site was used for cortical map reconstructions. C2 exclusive and overlapping cortical areas are depicted in the figure, as well as all exclusive cortical representational areas recorded in this experiment from one rat. Grey lines signal approximate position of rows $\mathrm{A}, \mathrm{B}, \mathrm{C}, \mathrm{D}$ and $\mathrm{E}$, for reference.

\section{Reconstruction of the PMBSF}

Reconstruction of the PMBSF "map" was done based on areas assigned to each recording site, using the polygons method of Voronoi. A matrix was made of polygonal areas bounded by dividing lines that were equidistant with the closest sampled sites, thus creating a network of polygons around the recording points. A typical example is shown in Figure 1.

Recording sites were made as near as the blood vessels allowed. We made an average of 170 microelectrode penetrations in each experiment, 135 were within the PMBSF while 45 that were outside, served to delimit the PMBSF. We point out that we are presenting results from arcs 1 to 4 , and only from rats in which we mapped the entire PMBSF, to have a global evaluation of their evoked activity. The method used allowed for a regular and complete sampling of the PMBSF. The sizes of the areas assigned to the recording sites averaged $0.045 \mathrm{~mm}^{2}$, they were very similar throughout the PMBSF $[\mathrm{F}(19,60)=433$; $\mathrm{p}<0.977]$ and did not differ among subjects $[\mathrm{F}(1,59)=1.177 ; \mathrm{p}<0.282]$. For an example see figure 1 . Using these areas associated to each recording site, we determined the total cortical representational area, the area of representational overlap (cortical territory shared with adjacent vibrissae) and exclusive representational area (sites in which only PV evoked a consistent response) for each vibrissa.

\section{RESULTS}

\section{Size of the CRs}

The size of the overlapping CRs areas of the vibrissae varied systematically across the PMBSF layer IV, with areas of greater size in the ventral rows. In contrast, the sizes of the exclusive CRs of the VPs were homogenous throughout the PMBSF. The exclusive and total cortical representational areas for each vibrissa are shown in Figure 2 . The average size of the total cortical representation of vibrissae was $0.4174 \mathrm{~mm}^{2}$ $(\mathrm{SD}=0,0256)$. These electrophysiologically 
defined areas for the vibrissae had different sizes $[F(19,60)=3.212 ; \quad \mathrm{p}<0.0001]$, following the same pattern in all the arcs, with different sizes across rows $[\mathrm{F}(4,59)=$ $12.527, \mathrm{p}<0.0001]$. Vibrissae located in the superior or dorsal rows (A and B) in the snout of the rat, had a smaller cortical representation than those located in ventral rows (D and E). Cortical representation of vibrissae of intermediate row $\mathrm{C}$ was not significantly different from that of other rows (Tukey: A vs B p<.994; A vs C $\mathrm{p}<0.027 ;$ AvsD $\mathrm{p}<0.0001 ; \mathrm{A}$ vs $\mathrm{E}$ $\mathrm{p}<0.0001 ; \mathrm{B}$ vs $\mathrm{C} \mathrm{p}<0.075 ; \mathrm{B}$ vs $\mathrm{D} \mathrm{p}<$ $0.0001 ; B$ vs $E \mathrm{p}<0.001 ; \mathrm{C}$ vs $\mathrm{D} \mathrm{p}<0.102 ; \mathrm{C}$ vs $\mathrm{E} p<0.441)$.

The described differences in size were due to zones of representational overlap and not to changes in the areas of PV-exclusive representation. Those exclusive areas, which in our data averaged $0.079 \mathrm{~mm}^{2}$ (SD
$=0.00757)$, did not differ significantly throughout the PMBSF $[\mathrm{F}(19,60)=0.469$; p<0.966] (Fig. 2). In comparison, the areas of representation that each vibrissa shared with others, or areas of representational overlap $\left(X=0.3386 \mathrm{~mm}^{2} ; \mathrm{SD}=0.02578\right)$, followed the same pattern that applied to the total area of representation. The overlapping areas for vibrissae in each row were closely similar, but were different between rows [Arcs: $F(3,59)=1.958$; $\mathrm{p}<0.718 ; \quad$ Rows: $\mathrm{F}(4,59)=10.134$; $\mathrm{p}<0.00001]$. The overlapping areas of cortical representation, as the total areas, were smaller in the dorsal than in the ventral rows (Tukey: A vs. B p $<0.998$; A vs. $\mathrm{C} p<0.243$; A vs. $\mathrm{D} p<0.002$; A vs. E $\mathrm{p}<$ 0.014 ; B versus $C \mathrm{p}<0.394$; B versus $\mathrm{D} p<$ 0.004 ; B vs. E $\mathrm{p}<0.032$; $\mathrm{C}$ versus $\mathrm{D}$ $\mathrm{p}<0.373$; $C$ vs. $E \mathrm{p}<0.777$; and $\mathrm{D}$ vs. $\mathrm{E}$ $\mathrm{p}<0.967)$.

\section{Exclusive and Total \\ Cortical Representational Areas}

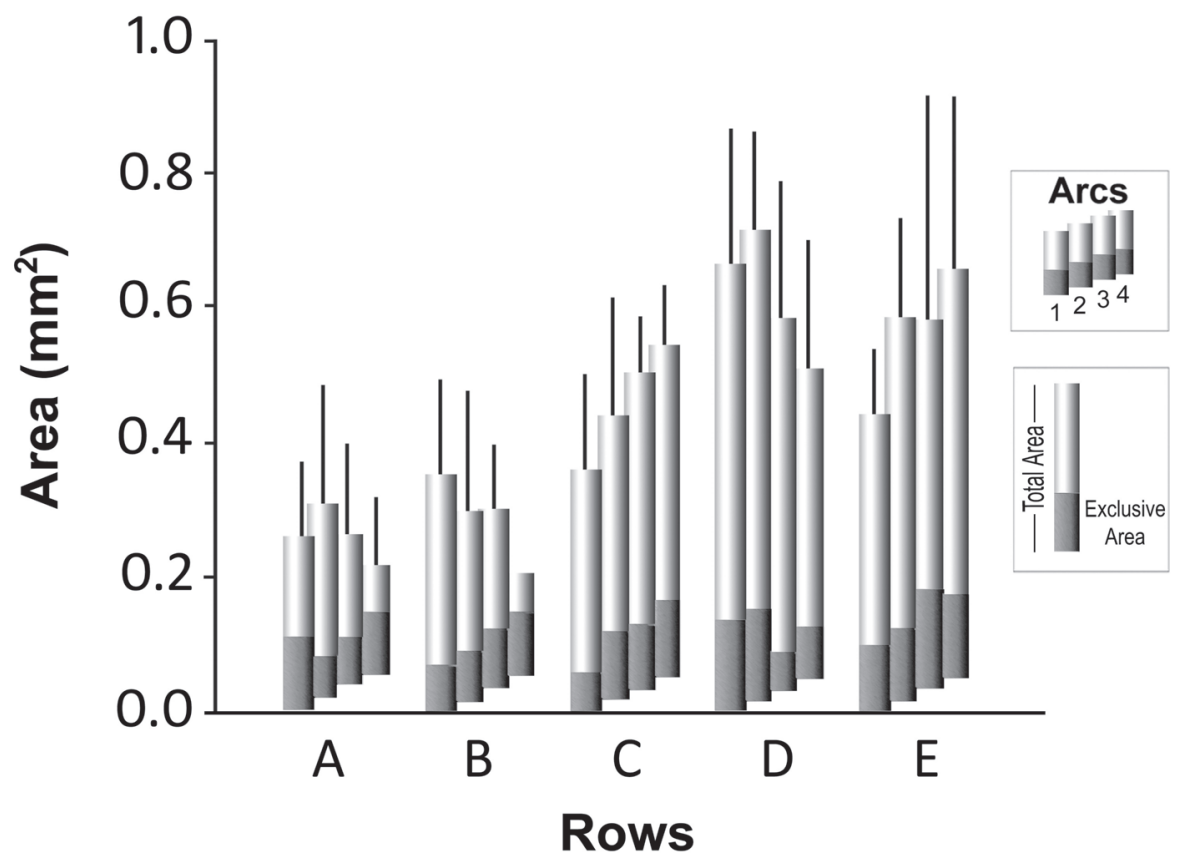

Figure 2: Exclusive and total cortical representational areas. The height of the bars represents the size of total cortical representational areas for each vibrissa. The bottom section of each bar represents the exclusive areas of cortical representation, both in $\mathrm{mm}^{2}$. The bars are located resembling the distribution of vibrissae and barrels in arcs 1-4. 
The total area of cortical representation of vibrissae was highly correlated with the overlapping area (Pearson, $r=0.878$; $\mathrm{p}<0.00001)$, but not with the area of exclusive representation (Pearson $r=0.207$; $\mathrm{p}=0.104)$.

Directional bias of overlapping areas and of $R F s$

The configuration of the vibrissae overlapping CRs and of the RFs of the cells of layer IV of the cortex has a bias, extending for greater distances along rows than arcs.

The cortical representation of vibrissae located in the same row were significantly more overlapped than that of vibrissae located in the same arc (row overlapping: $\mathrm{X}=0.3289 \mathrm{~mm}^{2}, \quad \mathrm{SD}=0.0290 ;$ Arc overlapping: $X=0.139 \mathrm{~mm}^{2}, \mathrm{SD}=0.014 ; \mathrm{t}$ $=8.705$, d.f. $=79 ; \mathrm{p}<0.0001)$. Of the total area of cortical representation that one vibrissa shared with others, $71.4 \%$ was with vibrissae in the same row, while only $28.6 \%$ was with vibrissae in the same arc; a difference that is highly significant $(\mathrm{t}=$ 9.288; d.f. $=79 ; \mathrm{p}<0.001)$.

These results demonstrate that the interaction between the cortical columns in layer IV of the PMBSF is not homogenous. In the normal PMBSF, anterior-posterior (intra-row) biases are predominant. Although the more ventral rows of vibrissae have greater areas of overlap than the dorsal rows, the direction of overlap is surprisingly constant over all the rows. Each vibrissa shares greater cortical area with others of its same row than with those of its same arc, independently of the size of the shared area.

As expected, the sizes of the receptive fields extended greater distances in the row-ward than in the arc-ward direction (Fig. 3). This classic measurement to study the sensory representation supports the idea that the spatial configuration of vibrissae, in rows and arcs, is functionally significant. The PV shared each cortical site with an average of 1.1516 adjacent vibrissae of its same row and with just 0.3832 vibrissae of its same arc $(\mathrm{t}=13.338$; d.f.=79; $p<0.0001)$.

\section{Participation of AV in Multiple-Vibrissae RFs}

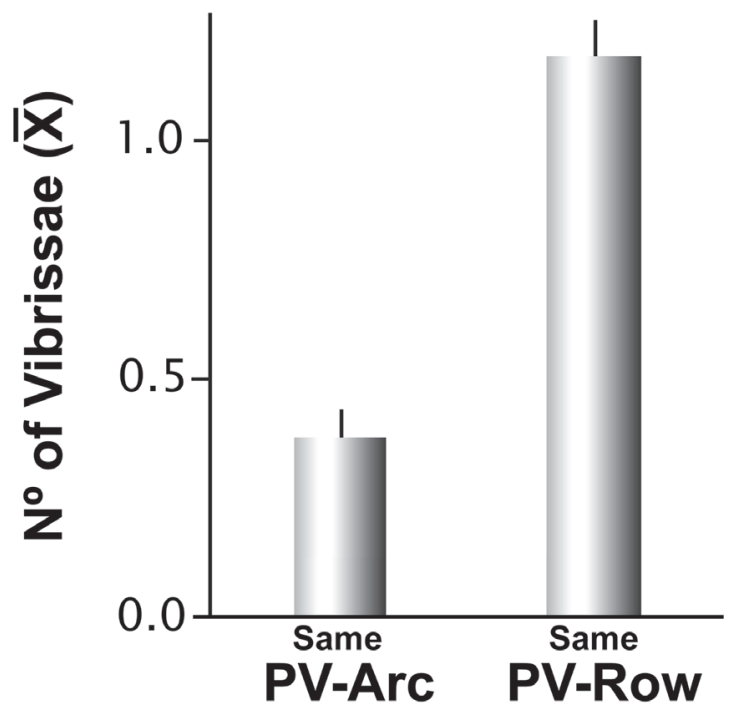

Figure 3: Participation of Adjacent Vibrissae (AV) in multiple-vibrissae RF. Average number of adjacent vibrissae located in the same arc or row as the principal vibrissae in multiplevibrissae RF.

These results imply that there is an asymmetry in the processing of information in the PMBSF between a cortical column and its neighbors, depending on whether they are in the same row or in the same arc.

\section{DISCUSSION}

The main contribution of this work is the revelation of a systematic heterogeneity and demonstration of sustained asymmetry among cortical columns in layer IV of the PMBSF. The barrel cortex has been viewed operationally as a conglomerate of essentially homogenous cortical columns that interact equivalently in all dimensions in layer IV. These simple reconstructions reported here show that this predominant view should be revised.

Studying the global operation of the PMBSF in the present work, we describe the following patterns that repeat themselves consistently throughout the PMBSF: A) Vibrissae have areas of $\mathrm{CR}$ of 
different sizes, depending on the row in which they are located. B) These differences are due to differences in areas in which there is overlap of the CRs of two or more vibrissae. C) The areas of exclusive representation of principal vibrissae have similar sizes throughout all the PMBSF. D) The areas of overlap and the configuration of the RFs both have a bias, in the rowward vs arc-ward direction. These results contribute to the understanding of the form in which the interaction between cortical columns takes place, at the level of layer IV of the somatosensory cortex of the rat.

Our finding becomes relevant because the vibrissal system is being used as a model for studying important subjects in neuroscience, such as the flow of the information processing in the sensory cortex (e.g. Brecht, 2007; Diamond et al, 2003; Miller et al., 2001; Moore et al., 1999; Wilent and Contreras, 2004), the relations between thalamus and cortex (e.g. Fox et al., 2003; Jones 2000; Kelly et al., 1999; Kwegyir-Afful et al, 2005) and neuronal cortical plasticity in relation with memory and learning (e.g. Berger et al 2007; Crochet and Petersen, 2006; Diamond et al 1999; Harris et al., 2001; Petersen, 2007; Polley et al, 2004).

General aspects: Exclusive and overlapping areas of cortical representation

In agreement with classic works (Armstrong-James et al., 1991; Simons, 1978; Welker, 1971; Welker et al., 1989; Woolsey \& Van Der Loos, 1970), our results show that:

1. The principal vibrissae of the RFs of neurons of layer IV of the PMBSF reproduce the spatial distribution of vibrissae in the sensorial periphery. However, there are also larger RFs that include other vibrissae in addition to the PV.

2. Data from 80 barrels (rows $A$ to $E$ in arc 1 to 4 from 4 rats) showed an average area of PV-exclusive representation of 0.08 $\mathrm{mm}^{2}$, which according to anatomical data, corresponds to the size of a barrel (about $0.09 \mathrm{~mm}^{2}$, e.g Welker et al., 1989).
3. The total area of the CR measured for each vibrissa was about $0.4 \mathrm{~mm}^{2}$. This value is in agreement with estimates from the classic works of Welker (1971), in which the cortical representation of vibrissae fluctuated between 0.6 and 0.3 $\mathrm{mm}^{2}$. This area of $0.4 \mathrm{~mm}^{2}$ would include, in addition to the area of exclusive representation, the septa that surround the barrel corresponding to the principal vibrissa and adjacent barrels, which belong to other principal vibrissae. Septa located between barrels of a row are smaller than septa between barrels of a column (Woolsey \& Van Der Loos, 1970). Therefore our data that show greater areas of overlapping of CRs within rows than within arcs cannot be explained by the size of septa.

The method of recording and data analysis, used in the present work, made it possible to study the operation of the PMBSF from a global perspective. We maximized the care of the preparation, to obtain a detailed study of the RFs of all the PMBSF. We strictly respected blood vessels, therefore maintaining the irrigation to the entire cortical area, which among other things, allowed us to keep the preparation in good physiological conditions throughout the recording period. In spite of this advantage, which allowed us to make an extensive mapping of the evoked activity in the PMBSF, in our work unlike others (Diamond et al., 1993, 1994, 1999; Moore et al., 1999; Moore \& Schady, 2000) the activity was not studied as a function of time. Consequently, our data do not contribute to the understanding of the flow of information over time, but they do offer a careful and exhaustive spatial analysis.

Besides covering all of the PMBSF, the studied cortical area was evenly sampled, allowing us to properly evaluate the horizontal distribution of evoked activities. The area associated with each recording site was on average $0.045 \mathrm{~mm}^{2}$. This corresponds to approximately 10 electrode penetrations to determine the total cortical representation of each vibrissa. The variability of these sampling areas was very 
low throughout the PMBSF of these intensively studied rats.

We are quite confident that our recordings were done in layer IV, because in numerous previous papers 750 microns under the duramater corresponded to this cortical layer (e.g Armstrong-James et al., 1991; Simons, 1978; Welker, 1971; Welker et al., 1989; Woolsey \& Van Der Loos, 1970). On the other hand, our histological control allowed us to estimate the vertical and horizontal positioning of small electrolytic micro lesions done in each rat after the recordings. However, vertical positioning is difficult to derive from flattened cortex preparation and we do not have the data to assure that all the sites we recorded were from layer IV.

\section{Origin of multi-vibrissa $C R$ in layer IV of the cortex}

The activity evoked by more than one vibrissa in the same cell necessitates input convergence. It has been proposed that this convergence occurs in layer IV, through horizontal connections among different cortical columns (Armstrong-James et al., 1991; Fox, 1994). However, Simons (1989) shows evidences supporting the idea that thalamo-cortical afferents go to more than one barrel.

Even though there is no consensus as to which of the aforementioned mechanisms would explain the overlap of two or more vibrissae CRs that takes place in layer IV, the evidence favors the role of thalamic afferents (Goldreich et al., 1999; Petersen $\&$ Diamond, 2000). For later stages of cortical processing, in supra and infra granular layers, there is agreement about its cortical origin.

Horizontal connections among columns in cortical layer II-III (Bernardo et al., 1990) along with the existence of pyramidal cells of layer IV, could explain the multivibrissa RFs in the supra and infra-granular layers. The apical dendrite of these cells would make contact with the horizontal connections existing between columns at the level of layers II and III, which could in turn affect the activity of layer IV. Thus a feedback to layer IV, through the non- granular cells could explain an increase of the area of representation of a vibrissa in layer IV. We cannot discard this possibility for explaining the multi-vibrissa RFs obtained at the level of layer IV. Since we did not measure responses in time, we were not able to differentiate the responses components from short-latency thalamic afferents from long-latency cortico-cortical afferents.

\section{Heterogeneity and asymmetry in the PMBSF}

Sizes of cortical representational areas of all vibrissae showed that while they were non-homogenous, they followed a certain pattern that was repeated throughout the PMBSF.

This spatial pattern was attributable to consistent proportional differences in areas of overlap, and not to variation in the areas of exclusive CR for the principal vibrissae. The latter were surprisingly constant all across the PMBSF. The areas of CR dedicated to the PVs and the areas in which the CRs of several vibrissae coincide, follow different spatial patterns throughout the PMBSF. This differentiation is consistent with the theory of parallel processing, as stated by Ahissar (Ahissar et al., 2000; Brumberg et al., 1999).

As we know, the PVs evoke exclusive responses mainly in cells located in the barrels, while cells with multi-vibrissa RFs are located generally in the septa. In addition, it is known that in the lemniscal system afferents from vibrissae to the posteroventral nucleus of the thalamus end in the barrels of layer IV, while in the extra-lemniscal system, afferents from the medial portion of the posterior nucleus end, among other sites, in the septa between barrels of layer IV. It is then reasonable to relate the lemniscal system that would be in charge of the discrimination of objects to a somatotopically ordered homogenous pattern of areas of exclusive representation of each vibrissa that would allow for better discrimination of the form and size of objects. On the other hand, the extralemniscal system, that would process information about the position of objects, 
may be related with the cells displaying approximately 5 times larger multi-vibrissa receptive fields.

We want to emphasize that although the areas of overlap between vibrissae of a same row have equal size, the areas from different rows differ in size. We hypothesize that this evidence suggests that the rows constitute specialized subgroups to detect certain ranks of some of the dimensions of sensorial event, possibly related to detection or location of objects in space.

In addition, our results show that the interaction of a column with its neighbors is asymmetric. Through all the PMBSF, the shape of the CR areas that one vibrissa shares with others is larger in rows than in arcs. One vibrissa shares approximately $70 \%$ of its cortical territory with other vibrissae of its row and $30 \%$, with other vibrissae of its arc. This relation remains constant throughout the PMBSF and is independent of the sizes of CR areas, which vary substantially from row to row. We postulate that this bias in the CR could be determined by the history of stimulation. The fact that a row constitutes a more homogenous unit than an arc could be interpreted as the result of the activity originating in the sensory periphery. The activity of each row is highly synchronized, due to the presence of muscles that connect the follicles of each row that are responsible for their exploratory movements. Based on works that demonstrate experience-dependent reorganization of the maps of cortical representation (Buonomano \& Merzenich, 1998; Merzenich et al., 1983; Jenkins et al., 1990; Guic \& Merzenich, 1990), we expect that the above mentioned characteristics of the receptors determine homogeneity between the elements of a row but not between those of a column.

The present work explores, for the first time, the existence of a preferred direction of the RFs and of the areas of cortical overlap, although in earlier reports the subject has been mentioned (e.g. Simons, 1978). Nevertheless, due to the lack of a systematic approach, even recent works assume a symmetrical relationship between neighboring columns (Armstrong-James, 1992; Ferezou et al., 2007; Petersen, 2007; Petersen \& Diamond, 2000; Shimegi et al., 2000). However, at a behavioral level there has been a differentiation between rows and arcs; Harris et al. (2001) demonstrated that the transference between two vibrissae in the execution of a detection task was greater when both were in the same row, rather than in the same arc. This result may depend on the characteristics of the task. In a previous study in our laboratory, rats learned to discriminate two degrees of roughness faster with only 3 vibrissae left in an arc rather than with 3 vibrissae left in a row (Guic-Robles et al., 1989). We postulate that the greater or smaller overlaps may be advantageous, depending on the specific behavioral challenges in the task.

Our results allow us to postulate that in the vibrissa-barrel sensory system, each vibrissa may be regarded as participating in two subsystems, distinguished by its interactions with other vibrissae in its row or arc. The row subsystem is characterized by its elements having a greater overlap in the cortex, occupying a proportionally larger cortical territory, by more extended receptive fields. These characteristics were reversed when the vibrissae were analyzed as a part of the arc subsystem. We postulated that rows are best equipped for greater somatosensorial sensitivity, while arcs are better equipped for spatial sharpness.

\section{ACKNOWLEDGMENTS}

We dedicate this work to our friend and mentor Teresa Pinto-Hamuy, who enthusiastically supported this research.

Supported by DIUC \#91038 and FONDECYT \#1930721 to E. Guic-Robles.

\section{REFERENCES}

AHISSAR E, SOSNIK R, HAIDARLIU S (2000) Transformation from temporal to rate coding in a somatosensory thalamocortical pathway. Nature 406: 302-306

ARMSTRONG-JAMES M, FOX K (1987) Spatiotemporal 
convergence and divergence in the rat S1 "barrel" cortex. The Journal of Comparative Neurology 263: 265-281

ARMSTRONG-JAMES M, CALLAHAN C, FRIEDMAN $M$ (1991) Thalamocortical mechanisms in the formation of receptive field of the rat barrel cortex neurons intracortical mechanisms. Journal of Comparative Neurology 303: 193-210

ATSUYUKI O, BARRY B, KOKICHI S SUNG N (2000) Spatial Tessellations - Concepts And Applications Of Voronoi Diagrams. 2ND EDITION John Wiley

BERGER T, BORGDORFF AJ, CROCHET S, NEUBAUER FB, LEFORT S, FAUBET B, FEREZOU I, CARLETON A, LUSCHER HR AND PETERSEN CCH (2007) Combined voltage and calcium epifluorescence imagingin vitro and in vivo reveals subthreshold and suprathreshold dynamics of mouse barrel cortex Journal of Neurophysiology 97: 3751-3762

BERNARDO KE, MCCASLAND JS, WOOLSEY TA, STROMINGER RN (1990) Local intra and interlaminar connections in mouse barrel cortex. The Journal of Comparative Neurology 291: 231-255

BRECHT M (2007) Barrel cortex and whisker-mediated behaviors. Current Opinion in Neurobiology 17: 408416

BRUMBERG JC, PINTO DJ, SIMONS S (1999) Cortical columnar processing in the rat whisker-to-barrel system. Journal of Neurophysiology 82: 1808-1817

BUONOMANO DV, MERZENICH MM (1998) Cortical plasticity: from synapse to maps. Annual Review of Neuroscience 21: 149-186

CROCHET S AND PETERSEN CCH (2006) Correlating whisker behavior with membrane potential in barrel cortex of awake mice. Nature Neuroscience 9: 608-610

DIAMOND ME, ARMSTRONG-JAMES M, EBNER FF (1993) Experience-dependent plasticity in adult rat barrel cortex. Proceedings of the National Academy of Sciences USA 90: 2082-2086

DIAMOND ME, HUANG W, EBNER FF (1994) Laminar comparison of somatosensory cortical plasticity. Science 265: 1885-1888

DIAMOND ME, PETERSEN RS, HARRIS JA (1999) Learning through maps: functional significance of topographic organization in primary sensory cortex. Journal of Neurobiology 41: 64-68

DIAMOND ME, PETERSEN RS, HARRIS JA AND PANZERI S (2003) investigations into the organization of information in sensory cortex. Journal of Physiology Paris 97: 529-536

DÖRFL J (1982) The musculature of the mystacial vibrissae of the white mouse. Journal of Anatomy 135(1): 147-154

FEREZOU I, HAISS F, GENTET L, ARONOFF R, WEBER B, PETERSEN C (2007) Spatiotemporal dynamics of cortical sensoriomotor integration in behaving mice. Neuron 56: 907-923

FOX K (1994) The cortical component of experiencedependent synaptic plasticity in the barrel cortex. Journal of Neuroscience 14: 7665-7679

FOX K, GLAZEWSKI S, SCHULZE S (2000) Plasticity and stability of somatosensory maps in thalamus and cortex. Current Opinion in Neurobiology 10: 494-497

FOX, KEVIN; WRIGHT, NICHOLAS; WALLACE, HELEN; GLAZEWSKI (2003) The origin of cortical surround receptive field studied in the barrel cortex. The Journal of Neuroscience 23(23) 8380-8391

GOLDREICH D, KYRIAZI HT, SIMONS D (1999) Functional independence of layer IV barrel in rodent somatosensory cortex. Journal of Neurophysiology 82: 1311-1316
GUIC-ROBLES E, GUAJARDO G, VALDIVIESO C (1989) Rats can learn a roughness discrimination using only their vibrissae. Behavioral Brain Research 31: 285-289

GUIIC-ROBLES E, MERZENICH MM (1990) Functional reorganization of barrel cortex in adult rats after vibrissal discrimination learning. Perspectives of Neuroscience on South America IBRO- UNESCO

HARRIS JA, HARRIS IM, DIAMOND ME (2001) The tipography of tactile learning in humans. The Journal of Neuroscience 21(3): 1056-1061

HARRIS JA, PETERSEN RS, DIAMOND ME (2001) The cortical distribution of sensory memories. Neuron 30 : 315-318

ITO M, SEO ML (1983) Avoidance of neonatal cortical lesions by developing somatosensory barrels, Nature 30: $1600-602$

JENKINS WM, MERZENICH MM, OCHS MT, ALLARD T, GUIC-ROBLES E (1990) Functional reorganization of primary somatosensorv cortex in adult owl monkeys after behavioral controlled tactile stimulation. Journal of Neurophysiology 63 (1): 82-104

JONES E (2000) Cortical and sub-cortical contributions to activity-dependent plasticity in primate somatosensory cortex. Annual Review of Neuroscience 23: 1-37

KELLY MK, CARVELL GE, KODGER JM, SIMONS DJ (1999) Sensory loss by selected whisker removal produces immediate disinhibition in the somatosensory cortex of behaving rats. The Journal of Neuroscience 19(20): 9117-9125

KWEGYIR EE, BRUNO R, SIMONS D AND KELLER (2005) A The role of thalamic inputs in surround receptive fields of the barrel neurons. The journal of Neuroscience 25(25): 5926-5934

MERZENICH MM, KAAS J.H, WALL JT, SUR M, NELSON RJ, FEILEMAN DJ (1983a) Topographic reorganization of somatosensory cortical areas $3 \mathrm{~b}$ and 1 in adult monkeys following restricted deafferentation. Neuroscience 8: 33-55

MERZENICH MM, KAAS JH, WALL JT, SUR M, NELSON RJ, FELLEMAN DJ (1983b) Progression of change following median nerve section in the cortical representation of the hand in areas $3 \mathrm{~b}$ and 1 in adult owl squirrel monkeys. Neuroscience 10: 639-665

MILLER KD, PINTO DJ, SIMONS DJ (2001) Processing in layer 4 of the neocortical circuit: new insights from visual and somatosensory cortex. Current Opinion in Neurobiology 11: 488-497

MOORE CI, NELSON S, DUR M (1999) Dynamics of the neural processing in rat somatosensory cortex. Trends in Neuroscience 22: 513-520

MOORE CEG, SCHADY W (2000) Investigation of the functional correlates of reorganization within the human somatosensory cortex. Brain 123: 1883-1895

PETERSEN C (2007) The functional organization of barrel cortex. Neuron 56: 339-355

PETERSEN RS, DIAMOND ME (2000) Spatial-Temporal distribution of whisker-evoked activity in the rat somatosensory cortex and the coding of stimulus location. The Journal of Neuroscience 20(16): 61356143

POLLEY, DANIEL; KVASNAK, EUGEN AND FROSTIG, RON. Naturalistic experience transforms sensory maps in the adult cortex of caged animals Nature 429: 67-71, 2004

POLLEY D, STEINBERG E, MERZENICH M (2006) Perceptual learning directs auditory cortical map reorganization through top-down influences. The journal of neuroscience 26(18): 4970-4982

SHIMEGI S, AKASAKI T, ICHIKAWA T, SATO H 
(2000) Physiological and anatomical organization of mutiwhisker response interaction in the barrel cortex of rats. The Journal of Neuroscience 20 (16): 6241-6248

SIMONS DJ (1978) Response properties of vibrissa units in rats SI somatosensory neocortex. Journal of Neurophysiology 41: 798-820

SIMONS DJ (1983) Multiwhisker stimulation and its effects on vibrissae units in the rat SMI barrel cortex. Brain Research 276: 178-182

WELKER C (1971) Microelectrode delineation of fine grain somatotopic organization of SMI cerebral neocortex in albino rat. Brain Research 26: 259-275

WELKER E, SORIANO E, VANDERLOOS H (1989) Plasticity in the barrel cortex of the adult mouse: effects of peripheral deprivation on gad- immunoreactivity. Experimental Brain Research 74: $441-452$

WILENT WB AND CONTRERAS D (2004) Synaptic responses to whisker deflections in rat barrel cortex as a function of cortical layer and stimulus intensity The Journal of Neuroscience 24(16): 3985-3998

WOOLSEY TA, VAN DER LOOS H (1970) The structural organization of layer IV in the somatosensory region (si) of mouse cerebral cortex: the description of cortical field composed of discrete cytoarchitectonic units. Brain Research 17: 205-249

WOOLSEY TA, WANN JR (1976) Areal changes in mouse cortical barrels following vibrissal damage at different post-natal ages. Journal of Comparative Neurology 170: $53-76$ 